\title{
ASYMPTOTIC AND CONVERGENT FACTORIAL SERIES IN THE SOLUTION OF LINEAR ORDINARY DIFFERENTIAL EQUATIONS ${ }^{1}$
}

ROBERT L. EVANS

For the differential equation

$$
\sum_{\beta=0}^{n} P_{\beta}(x) y^{(n-\beta)}=0
$$

where

$$
P_{\beta}(x)=\sum_{\mu=-m(\beta)}^{\infty} p_{\beta, \mu} x^{-\mu} \quad\left(p_{\beta, m(\beta)} \neq 0 \text { and } \beta=0,1, \cdots, n\right)
$$

converges for all $|x|>x_{0}$ (and $|x|>x_{0}$ contains no finite singular points of (1)), Fabry ${ }^{2}$ has presented $n$ linearly independent formal solutions about $x=\infty$. These are of the form

$$
y_{\nu}(x) \sim e^{Q(v, x)} \sum_{\mu=0}^{\infty} a_{\nu, \mu} x^{\rho(\nu)-\mu / m} \quad(\nu=1,2, \cdots, n)
$$

where $Q(\nu, x)$ is a polynomial in $x^{1 / m}$ and the series are generally asymptotic but not convergent. For special cases of (1), Horn ${ }^{3}$ and Trjitzinsky ${ }^{4}$ have obtained solutions in which the asymptotic series are replaced by convergent series of inverse factorials. This development is extended here to all cases of (1), which then makes it possible to solve "in the large" those cases of (1) in which the $P_{\beta}$ 's are polynomials.

The Puiseux diagram and integer $m$. The Puiseux diagram for (1) about ${ }^{5} x=\infty$ is the highest convex-upward polygonal line having all its vertices at points $(\beta, m(\beta))(\beta=0,1, \cdots, n)$. The rank of (1) at $x=\infty$ is defined to be 1 unit greater than the greatest slope on this dia-

Presented in part to the Society, April 28, 1951; received by the editors November 14, 1952 and, in revised form, June 19, 1953.

${ }^{1}$ Part of this material is from the author's thesis under the encouraging and helpful advisorship of Professor H. L. Turrittin. The manuscript preparation was aided by Office of Ordnance Research project DA-11-022-ord 489, in which these results can be used.

2 E. Fabry, These, Paris, 1885.

${ }^{3}$ J. Horn, Math. Zeit. vol. 21 (1924) pp. 85-95.

${ }^{4}$ W. J. Trjitzinsky, Trans. Amer. Math. Soc. vol. 37 (1935) pp. 80-146.

5 E. L. Ince, Ordinary differential equations, New York, Dover, 1944, p. 298 for the Puiseux diagram about $x=0$. 
gram. If the rank exceeds $0, x=\infty$ is an irregular singular point of (1). If one makes the substitution

$$
x=z^{m}
$$

in (1), (2), and (3), the fractional powers can be removed from the "solutions," (3). It is possible to find a single value for $m$ in (4) that will assure the removal of fractional powers from all $n$ "solutions" in (3). For this, it is sufficient in all cases of (1) (and necessary in some) to set $m=1$.c.m. $\left(1,2, \cdots, \beta_{0}\right)$, where $\beta_{0}$ is the greatest number of $\beta$ spaces that are covered by any one of the segments of the Puiseux diagram. This value of $m$ will also change the Puiseux slopes to integers. If one of the new Puiseux slopes is 0 we double the above $m$ value in order to make all those slopes either $>0$ or $\leqq-1$, for a purpose to be described.

\section{Let}

$$
y_{\nu}\left(z^{m}\right)=z^{m \cdot \rho(\nu)+1} \cdot v_{\nu}(z) \exp \left(Q\left(\nu, z^{m}\right)\right)
$$

define $v_{\nu}(z)$. Next consider a particular $v_{\nu}(z)$, but with omission of the index for convenience. If this $v(z)$ is related to a Puiseux slope of $s_{0}$ on the diagram for (1), it is related to a Puiseux slope of $m\left(s_{0}+1\right)-1$ after the substitution of (4). Then $a_{v, \mu}=a_{\mu}$ is of the order $O\left(M^{\mu}(\mu !)^{1-\delta}\right)$ (where $M$ is a fixed positive number and $\delta=1-1 / m\left(s_{0}+1\right)$ ) uniformly with respect to $\mu=0,1, \ldots .6$ Our $m$ is chosen large enough to make $\delta>0$. The solutions and cases of (1) which were treated by Horn ${ }^{3}$ and Trjitzinsky ${ }^{4}$ relate to the first or left-most segment on the Puiseux diagram and have $\delta=0$.

In these details it is assumed for simplicity and without loss of generality that $v(z)$ is not a solution containing $\log z .^{7}$

Laplace integral and factorial series solutions. The $v(z)$ of each solution, $y(z)$, can be represented by a factorial series whose convergence is to be demonstrated. The factorial series is most easily obtained from the asymptotic series in (5), and the proof of its convergence utilizes a related Laplace integral.

Define $V_{\nu}(z)$ by the equation

$$
V_{\nu}(z)=a_{\nu, 0}+\sum_{s=0}^{\infty} \frac{b_{\nu, s} !}{z(z+\omega) \cdots(z+s \omega)}
$$

${ }^{6}$ L. M. Milne-Thomson, The calculus of finite differences, London, Macmillan, 1933 , chap. XV, which applies to the recurrence relation satisfied by the $a_{\mu}$ 's.

${ }^{7}$ When $\log z$ is involved, two or more solutions are interrelated and the recurrence relations for some of the $a_{r, \mu}$ 's are nonhomogeneous-but the order of magnitude of successive $a_{r, \mu}$ 's is unchanged. Therefore the eventual results apply to those cases too. 
where, in accordance with a result of Norlund, ${ }^{8}$

$$
b_{r, 8} \cdot s !=\sum_{k=1}^{\dot{1}} A_{k, 8}(\omega)^{-k} a_{v, k+1}(\text { if } s \geqq 1) \text { and } b_{v, 0}=a_{r, 1},
$$

in which $A_{0,8}=A_{k, k-1}=0, \quad A_{1,1}=1, \quad$ and $A_{k, 8}=(s-1) A_{k,(8-1)}$ $+A_{(k-1),(s-1)}\left(\right.$ if $s \geqq 2$ ), and where $|\omega|=1$. These $A_{k, 8}$ 's are the absolute values of Stirling's numbers of the first kind. ${ }^{9}$ The value of $m$ was chosen large enough to assure convergence (to be proved) of the factorial series in (6) and thus also the equality of $z \cdot v_{\nu}(z)$ and $V_{\nu}(z)$.

To prove the convergence of the factorial series in (6), consider the Laplace integral

$$
z \cdot v_{\nu}(z)=\int_{-\infty}^{0} e^{\xi z / \omega} \psi_{\nu}(\xi) d \xi
$$

where $^{10} \psi_{\nu}(\xi)=A_{0}+A_{1} \xi+A_{2} \xi^{2}+\cdots$, and $A_{\mu}=(-1)^{\mu} a_{\nu, \mu+1} /\left(\omega^{\mu+1}\right.$ $\cdot \Gamma(\mu+1))$, is of the order $O\left(M^{\mu}(\Gamma(\mu))^{-\delta}\right)$.

To relate the Laplace integral in (8) to the factorial series of (6), and to establish convergence, substitute

$$
\xi=\log t
$$

in (8) so that

$$
v_{\nu}(z)=\int_{0}^{1} t^{z / \omega-1} \psi_{\nu}(\log t) d t
$$

Further, $\psi_{\nu}(\log t)$ can be expanded in a series of powers of $(1-t)$ and this series will have unit radius of convergence because of the growth condition on the $A_{\mu}$ 's. For $R(z / \omega)>z_{0}$, and $z_{0}=\max \left(1, x_{0}^{1 / m}\right)$, the indeterminate form $t^{z / \omega-1} \cdot \psi_{\nu}(\log t)$ converges to 0 as $t \rightarrow 0$, so the integrand in (9) is uniformly convergent in $0 \leqq t \leqq 1$. This is a sufficient condition for convergence in (10) whenever $|z|>z_{0}$ and $|\arg z-\arg \omega| \leqq \pi / 2 .{ }^{11}$ The argument of $\omega$ is arbitrary. Furthermore, one can obtain the series in (6) by expanding $\psi_{\nu}(\log t)$ in powers of

N. E. Norlund, Vorlesungen über Differenzenrechnung, Berlin, Springer, 1924, p. 261.

- C. Jordan, Calculus of finite differences, New York, Chelsea, 2d ed., 1947, pp. 143-144 and pp. 160-162.

${ }^{10} \mathrm{E}$. L. Ince, footnote 5, pp. 443-445. Ince's restriction to differential equations of rank 1 is needed for getting all $n$ solutions but not in treating the single solution considered here, for which the exponential factor has already been accounted. Also, the contour of integration in (8) is appropriate to Ince's special cases and to the present special case.

11 Footnote 6, pp. 288-290. 
$(1-t)$ and integrating the right-hand side of (9) term by term. ${ }^{11}$ The final result of this section is thus:

Theorem 1. For $|z|>z_{0}$ and $|\arg z-\arg \omega| \leqq \pi / 2$, with arbitrary argument of $\omega$,

$$
V_{\nu}(z)=z \cdot v_{\nu}(z)
$$

and therefore equations (4) through (7), with (10), provide a solution of (1) and (2). The involved factorial series converges whenever $|x|$ $>\max \left(1, x_{0}\right)$ and $|\arg x-m \arg \omega| \leqq m \pi / 2$.

Since this solution is any one of the $n$ solutions of (1) and (2), the result applies to all $n$ solutions ${ }^{7}$ - which correspond to $\nu=1,2, \cdots, n$.

Accuracy estimate for the asymptotic representations. It follows that the accuracy of the asymptotic representation in (3) may be estimated by comparing it with the convergent factorial series solution, (4) through (7), plus (10). This estimate is given by, from (3) through (7), $\left|z \cdot v_{\nu}(z)-\sum_{\mu=0}^{k} a_{\nu, u} z^{-\mu}\right|=\mid \sum_{s=k}^{\infty}\left(\sum_{k=k}^{s} A_{k, s}(\omega)^{s-k}\right.$ $\left.\cdot a_{r, k+1}\right) / \prod_{k=0}^{s}(z+\mu \omega)$, where $k$ is an arbitrary positive integer.

University of Minnesota 